\title{
Selección de una wavelet madre para el análisis frecuencial de señales eléctricas transitorias usando WPD
}

\author{
Selection of a mother wavelet for frequency analysis \\ of transient electrical signals using WPD \\ $\begin{array}{lll}\text { E. Gómez-Luna } & \text { D. Silva } & \text { G. Aponte } \\ & \end{array}$ \\ Recibido: 30 de julio de 2012, aceptado: 11 de marzo de 2013 \\ Received: July 30, 2012, accepted: March 11, 2013
}

\begin{abstract}
RESUMEN
Este artículo propone un método para seleccionar una wavelet madre adecuada para el análisis frecuencial de señales eléctricas transitorias, considerando sus propiedades y las necesidades del caso de aplicación. Se utilizó la descomposición wavelet por paquetes analizando las wavelets como filtros y para cuantificar la dispersión de información evaluando el desempeño de diferentes familias incluidas en las librerías de Matlab. Para validar el método propuesto se presenta un caso de aplicación donde se estima el valor eficaz (RMS) de una señal transitoria.

El método de selección utilizado permite tener una visión clara de cómo aprovechar las propiedades de las wavelets para escoger una adecuada, dependiendo del caso de aplicación.
\end{abstract}

Palabras clave: Transformada wavelet, señales transitorias, wavelet madre, descomposición wavelet por paquetes, análisis frecuencial.

\section{ABSTRACT}

This paper proposes a method to select an appropriate mother wavelet for the frequency analysis of electrical transient signals, based on the wavelet properties and considering the needs of the application case. The Wavelet Packet Decomposition (WPD) was used to analyze wavelets as filters and to quantify the information leakage by evaluating the performance of different families included in the Matlab's libraries. To validate the method proposed, an application case is presented where the effective value (RMS) is estimated for a transient signal.

The selection method used provides a clear scope on how to exploit the wavelet properties to select the appropriate one depending on the particular application.

Keywords: Wavelet transform, transient signals, mother wavelet, wavelet packet decomposition, frequency analysis.

\section{INTRODUCCIÓN}

El análisis por Fourier ha sido una de las herramientas más importantes en el tratamiento de señales eléctricas, pero presenta inconvenientes cuando se requiere el estudio de señales no estacionarias, las cuales aparecen en el campo de la ingeniería habitualmente. Recientemente, la Transformada Wavelet (WT) ha tomado fuerza en el análisis de eventos transitorios debido a su propiedad de resolución variable en términos del tiempo y la frecuencia, lo que permite modificar el ancho de la ventana a aplicar, haciendo posible localizar en el tiempo los eventos de baja y alta frecuencia

1 Grupo de Investigación en Alta Tensión. Universidad del Valle. Calle 13 \#100-00, edificio 356, AA 25360. Santiago de Cali, Colombia.E-mail: eduardo.gomez@correounivalle.edu.co; diegofdosilva@gmail.com; guillermo.aponte@ correounivalle.edu.co 
simultáneamente [1-6]. Lo anterior permite analizar con buena resolución en frecuencia componentes que oscilan lentamente, tales como la frecuencia natural del sistema $(60 \mathrm{~Hz})$, sus armónicos y sus subarmónicos. También permite analizar con buena resolución en tiempo los componentes que oscilan rápidamente, que normalmente corresponden a eventos transitorios.

La calidad de la información obtenida a partir de un análisis donde se aplica la WT depende en gran medida de la wavelet madre utilizada, de modo que se debe considerar como una etapa dentro del análisis por WT la selección de una wavelet madre adecuada para cada caso particular de estudio [7-8].

En la bibliografía revisada no se identificó un método o algoritmo claro que permita la selección adecuada de una wavelet basado en sus propiedades, sino que básicamente se emplean tres criterios: el primero consiste en determinar visualmente la semejanza que tiene la forma de la wavelet madre y la de la señal bajo análisis; el segundo consiste en ensayar distintas wavelets o métodos para cada caso de estudio [9, 11], y a partir de los resultados escoger una; el último simplemente usa la wavelet db4, por ser la más empleada en el análisis de señales eléctricas [12-14].

Sin embargo, se dispone de una gran variedad de wavelets madre y cada una tiene características propias, por lo que pueden ofrecer diferentes resultados para cada caso de estudio particular.

\section{ANÁLISIS WAVELET}

La base de la Transformada de Fourier (FT) son funciones seno y coseno, añadiéndose para el caso de la Transformada en Ventana de Fourier (STFT) una función ventana para analizar por partes una señal determinada. Con la WT se conjugan estos dos conceptos de función base y función ventana, para resolver el problema de resolución del análisis por Fourier, el cual consiste en el compromiso entre resolución temporal y resolución frecuencial, tal como lo plantea el principio de incertidumbre de Heisenberg [15].

La transformación de la WT se realiza a través de una función llamada wavelet (o wavelet madre), con la que se descompone una señal en diferentes componentes de frecuencia que conforman una familia de funciones que son traslaciones y dilataciones de una función madre $\psi(t)$. Este proceso de traslación y dilatación se define en (1).

$$
\psi_{\tau, a}=\frac{1}{\sqrt{a}} \psi\left(\frac{t-\tau}{a}\right)
$$

Donde $\tau$ realiza la traslación y a provee la dilatación (o escalamiento).

Las wavelets deben tener valor promedio nulo, energía finita y cumplir con una condición de admisibilidad, estos tres criterios se muestran en las ecuaciones (2), (3) y (4), respectivamente.

$$
\begin{gathered}
\int_{-\infty}^{\infty} \psi(t) d t=0 \\
\int_{-\infty}^{\infty}|\psi(t)|^{2} d t<\infty \\
C_{\psi}=\frac{1}{\sqrt{2 \pi}} \int_{-\infty}^{\infty} \frac{|\bar{\psi}(\omega)|^{2}}{|\omega|} d \omega<\infty
\end{gathered}
$$

Donde $\widehat{\psi}(\omega)$ es la FT de $\psi(t)$.

La Transformada Wavelet Continua (CWT) se define en (5).

$$
C(\tau, a)=\frac{1}{\sqrt{a}} \int_{-\infty}^{\infty} x(t) \overline{\psi\left(\frac{t-\tau}{a}\right)} d t
$$

Donde $\overline{\psi(t)}$ es el complejo conjugado de $\psi(t)$.

Sin embargo, cuando se evalúa computacionalmente la CWT, los resultados son altamente redundantes debido a la gran cantidad de información, por esto se emplea la Transformada Wavelet Discreta (DWT), en la cual los parámetros $a$ y $t$ varían de acuerdo con (6) y (7), respectivamente.

$$
\begin{gathered}
a=a_{0}^{j} \\
\tau=k \tau_{0} a_{0}^{j}
\end{gathered}
$$

Donde $j$ es un entero positivo. 
Para mejorar la eficiencia en términos de cálculo computacional se toma $a_{0}=2$ y $\tau_{0}=1$, con esto se define la forma práctica de la DWT llamada Análisis Multirresolución (MRA), la cual puede ser vista como la descomposición de una señal usando filtros paso alto (HP) y paso bajo (LP), tal como se muestra en la Figura 1.

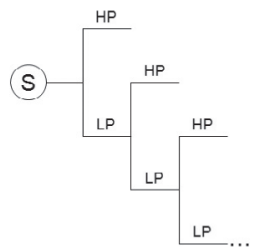

Figura 1. Árbol de descomposición del MRA.

Donde la salida de un filtro paso bajo se conoce como aproximación, y la salida de un filtro paso alto como detalle. En la Figura 2 se muestra cómo se divide el ancho de banda de la señal, a medida que se efectúa el proceso de descomposición.

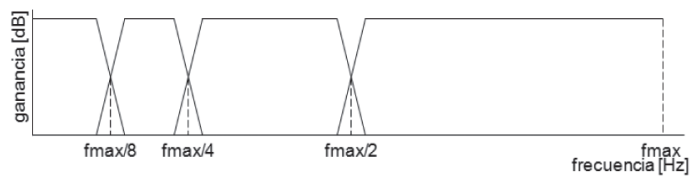

Figura 2. División del ancho de banda del MRA.

La división no uniforme del ancho de banda usando el análisis MRA hace que se concentre mucha información en los primeros componentes de frecuencia, lo cual puede ser un inconveniente dependiendo del tipo de aplicación, por tal motivo se hace la Descomposición Wavelet por Paquetes (WPD), dividiendo el ancho de banda de la señal de forma uniforme, tal como se presenta en la Figura 3.

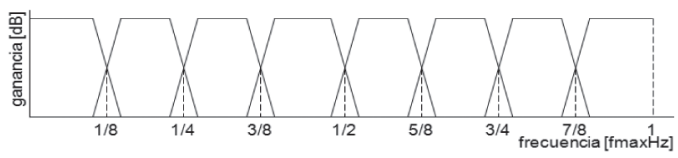

Figura 3. División del ancho de banda de la WPD.

Para lograr la división del ancho de banda usando la WPD se emplean etapas de filtrado tanto a las aproximaciones (información de baja frecuencia) como a los detalles (información de alta frecuencia), tal como se muestra en la Figura 4.

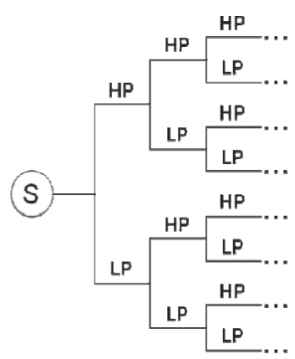

Figura 4. Árbol de descomposición de la WPD.

\section{CARACTERÍSTICAS Y PROPIEDADES DE LAS WAVELETS}

En la Tabla 1 se presentan las características de las wavelets más conocidas y algunas de sus propiedades. A continuación se presenta una descripción de las propiedades más importantes.

Momentos de desvanecimiento. Es una propiedad relacionada con la compresión de información y eliminación de ruido. El i-ésimo momento de desvanecimiento de la wavelet se calcula usando (8).

$$
\int_{-\infty}^{\infty} \psi(t) t^{i} d t=0
$$

De tal forma que la wavelet tiene $v$ momentos de desvanecimiento, si se cumple (8) para $i=0,1, \ldots, v-1$.

En términos prácticos, la cantidad de momentos de desvanecimiento está directamente relacionada con la selectividad de la descomposición wavelet, vista como proceso de filtrado [16].

Tamaño del soporte. Está directamente relacionado con la cantidad de momentos de desvanecimiento, debido a que en una wavelet con $v$ momentos de desvanecimiento el tamaño del soporte es $2 v-1$ [16]. Normalmente esta propiedad está directamente relacionada con la cantidad de coeficientes del filtro (teniendo en cuenta que una wavelet se puede ver como un filtro), e influye directamente en el tiempo de cálculo y en la distorsión que se produce en los extremos al procesar una señal; dado esto, aunque se prefiere una gran cantidad de momentos de desvanecimiento, se debe lograr un equilibrio entre ambos parámetros para llevar a cabo un análisis apropiado. 
Tabla 1. Características y propiedades de las wavelets más comunes.

\begin{tabular}{|c|c|c|c|c|c|c|c|c|c|c|c|}
\hline & Haar & $\begin{array}{c}\text { Mexican } \\
\text { Hat }\end{array}$ & Morlet & Daubechies & Symmlets & Coiflets & Gaussiana & Biorthogonal & $\begin{array}{c}\text { Reverse } \\
\text { Biorthogonal }\end{array}$ & Meyer & $\begin{array}{c}\text { Discrete } \\
\text { Meyer }\end{array}$ \\
\hline Propiedades & (haar) & (mexh) & (morl) & $(\mathrm{dbN})$ & (symN) & (coifN) & (gausN) & (biorNr.Nd) ${ }^{4}$ & $\left(\right.$ rbioNr.Nd) ${ }^{4}$ & (meyr) & (dmey) \\
\hline Orden & --- & --- & --- & $1,2, \ldots, 45^{2}$ & $1,2, \ldots, 41^{2}$ & $1,2, \ldots, 5^{2}$ & $1,2, \ldots, 44^{2}$ & $1.1,1.3, \ldots, 6.8^{2}$ & $1.1,1.3, \ldots, 6.8^{2}$ & --- & --- \\
\hline Regularidad & No & Sí & Sí & Relativa $^{3}$ & Relativa $^{3}$ & Relativa $^{3}$ & Sí & Relativa $^{3}$ & Relativa $^{3}$ & Sí & Sí \\
\hline $\begin{array}{l}\text { Tamaño } \\
\text { del Soporte }\end{array}$ & 1 & {$[-5,5]$} & {$[-4,4]$} & $2 \mathrm{~N}-1$ & $2 \mathrm{~N}-1$ & $6 \mathrm{~N}-1$ & {$[-5,5]$} & $2 \mathrm{Nd}+1$ & $2 \mathrm{Nr}+1$ & {$[-8,8]$} & {$[-8,8]$} \\
\hline $\begin{array}{l}\text { Longitud } \\
\text { del Filtro }\end{array}$ & 2 & -- & --- & $2 \mathrm{~N}$ & $2 \mathrm{~N}$ & $6 \mathrm{~N}$ & -- & $--^{6}$ & $--^{6}$ & --- & -- \\
\hline Simetría & Sí & Sí & Sí & No & Aproximada & Aproximada & Sí & Sí & Sí & Sí & Sí \\
\hline $\begin{array}{l}\text { Momentos de } \\
\text { Desvanecimiento }\end{array}$ & 1 & -- & --- & $\mathrm{N}$ & $\mathrm{N}$ & $2 \mathrm{~N}$ & -- & $\mathrm{Nr}$ & $\mathrm{Nd}$ & --- & --- \\
\hline $\begin{array}{l}\text { Función } \\
\text { de Escala }\end{array}$ & Sí & No & No & Sí & Sí & Sí & No & Sí & Sí & No & Sí \\
\hline CWT & Posible & Posible & Posible & Posible & Posible & Posible & Posible & Posible & Posible & Posible & Posible \\
\hline DWT & Posible & No Permite & No Permite & Posible & Posible & Posible & No Permite & Posible & Posible & No Permite & Posible \\
\hline $\begin{array}{l}\text { Expresión } \\
\text { Explíita }\end{array}$ & Sí & Sí & Sí & No & No & No & Sí & No & No & Sí & Sí \\
\hline
\end{tabular}

Regularidad. Es la capacidad de una wavelet de reconstruir fielmente una señal a partir los coeficientes calculados en el proceso de transformación [16], o dicho de otro modo, representa la suavidad de la wavelet.

Simetría. Si la wavelet es simétrica, al verla como un filtro se puede decir que tiene fase lineal, si no es simétrica se introduce distorsión en la fase [17]. Esto es de especial interés en aplicaciones de procesamiento de sonido e imágenes.

Función de escala. Es una función ortogonal a la wavelet y, eventualmente, si ambas funciones son normalizadas constituyen una base ortonormal del espacio [16]. Al no tener definida una función de escala, una wavelet determinada no puede ser utilizada en la aplicación de la DWT.

En síntesis, para el análisis frecuencial de señales transitorias las propiedades más importantes son: la cantidad de momentos de desvanecimiento, ya que es conveniente que los anchos de banda compartan el mínimo de información posible, y el tamaño del soporte, porque influye considerablemente en el tiempo de cálculo. También se debe considerar la existencia de la función de escala, ya que dependiendo del tipo de aplicación (CWT o DWT), algunas wavelets no podrán ser utilizadas. Sin embargo, la importancia de una propiedad sobre otra depende fundamentalmente de las necesidades del caso de aplicación.

2 Matemáticamente se podría tener un orden mayor al indicado, pero en Matlab las wavelets indicadas solo están definidas hasta dicho orden.

\section{LAS WAVELETS VISTAS COMO FILTROS}

Utilizando el teorema de Parseval [18] se puede visualizar la respuesta en frecuencia de las wavelets [19], para evidenciar la información que comparten los anchos de banda en el proceso de filtrado. Como ejercicio se puede aplicar la WPD a una señal senoidal que varía su frecuencia desde 0 hasta $1 \mathrm{kHz}$ (con frecuencia de muestreo $\mathrm{Fs}=2 \mathrm{kHz}$ ); para cada valor de frecuencia se calcula la energía que recoge cada componente en el nivel 2 de descomposición, con relación a la energía total de señal. En la Figura 5 y la Figura 6 se muestran las respuestas de las wavelets $\mathrm{db} 4 \mathrm{y} \mathrm{db} 20$, respectivamente.

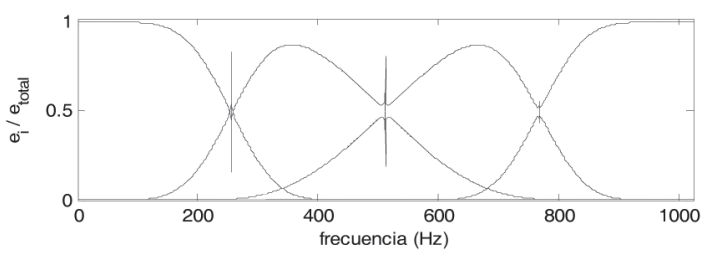

Figura 5. Respuesta en frecuencia de la wavelet $\mathrm{db} 4$.

\footnotetext{
3 La wavelet aumenta su regularidad en cuanto mayor es el orden.

4 Esta wavelet posee un orden compuesto por dos cifras y se debe a que utiliza una wavelet para el proceso de descomposición (Nd) y otra para el proceso de reconstrucción (Nr). No hay una secuencia para la combinación $\mathrm{Nr} . \mathrm{Nd}$, los posibles casos se pueden observar con el comando waveinfo( 'bior') o waveinfo('rbio') de MatLab.

5 Solo aplica para el proceso de descomposición.

6 No se puede dar una expresión, con el comando waveinfo( 'bior') o waveinfo('rbio') se pueden ver los valores.
} 


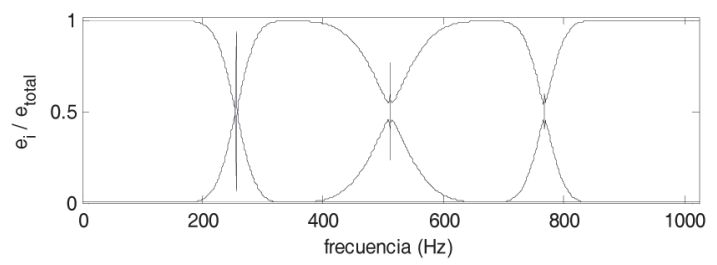

Figura 6. Respuesta en frecuencia de la wavelet $\mathrm{db} 20$.

Las dos figuras anteriores se pueden comparar con la Figura 3, donde se evidencia que el orden de la wavelet (asociado con la cantidad de momentos de desvanecimiento) influye sobre la dispersión de información frecuencial. Sin embargo, en este punto solo se muestra un análisis visual, que no es de gran ayuda si se quiere escoger una wavelet dentro de un grupo considerable; por tal motivo, en este trabajo se plantea un método que cuantifica la dispersión de información frecuencial logrando una cuantificación para tomar una decisión sobre la dispersión frecuencial de una wavelet.

\section{MÉTODO PARA CUANTIFICAR LA DISPERSIÓN}

El algoritmo planteado para cuantificar la dispersión que posee una wavelet determinada [20] se describe en la Figura 7, el cual consiste en aplicar la WPD y utilizar los componentes de cualquier nivel de descomposición, luego se determina cuánta energía queda por fuera del ancho de banda al que pertenece la frecuencia de la señal de entrada (el ancho de banda de la señal se determina con el teorema de Nyquist) y, por último, se promedian las energías de dispersión calculadas.

Cabe resaltar que se puede usar cualquier nivel de descomposición u otro rango para el barrido frecuencial, ya que los resultados se mantienen (esto fue probado haciendo un análisis de sensibilidad al algoritmo desarrollado), y la selección de los parámetros mencionados se basó principalmente en la resolución frecuencial y el tiempo de cálculo.

El método desarrollado se empleó en la selección de una wavelet aplicable al análisis de transitorios eléctricos, evaluando las cinco principales familias [16] disponibles en el Toolbox de MatLab:

- Daubechies (db1, 2, 3, .., 40).

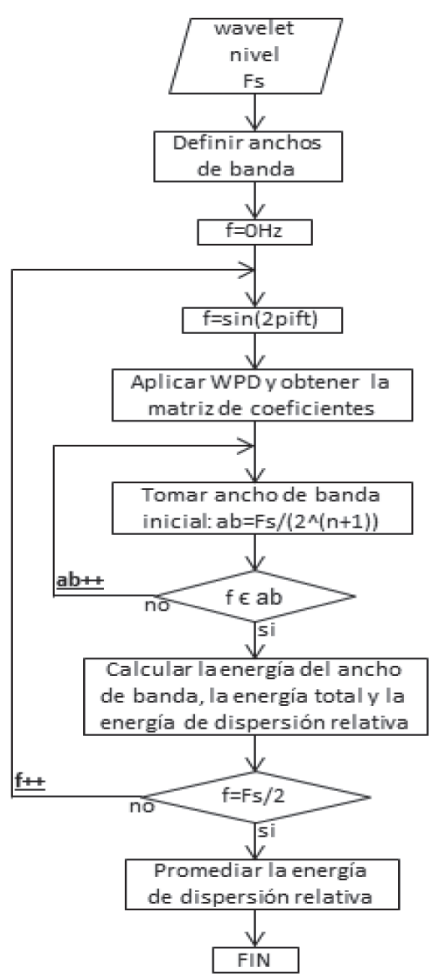

Figura 7. Algoritmo para cuantificar la dispersión.

- $\quad$ Symmlets (sym1, 2, 3, .., 25).

- Coiflets (coif1, 2, 3, 4, 5).

- Biorthogonal (bior1.1, 1.3, 1.5, 2.2, 2.4, 2.6, $2.8,3.1,3.3,3.5,3.7,3.9,4.4,5.5$ у 6.8 ).

- Reverse-Biorthognal (los mismos índices que la familia Biorthogonal).

El análisis se realizó sobre un total de 100 wavelets, mostrándose en la Figura 8 el resultado de evaluar la familia Daubechies.

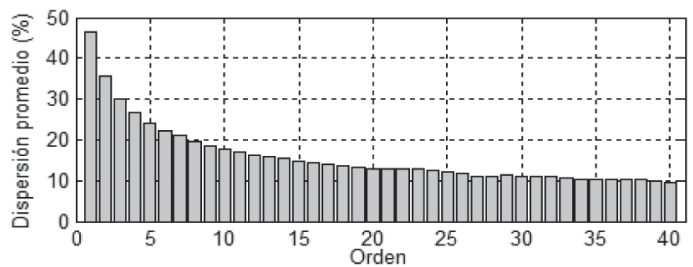

Figura 8. Dispersión promedio calculada con la familia Daubechies.

En el gráfico anterior se observa que al aumentar la cantidad de momentos de desvanecimiento disminuye la dispersión de información. 
En la Figura 9 se muestran los resultados obtenidos con todas las familias mencionadas, aplicando el mismo procedimiento.

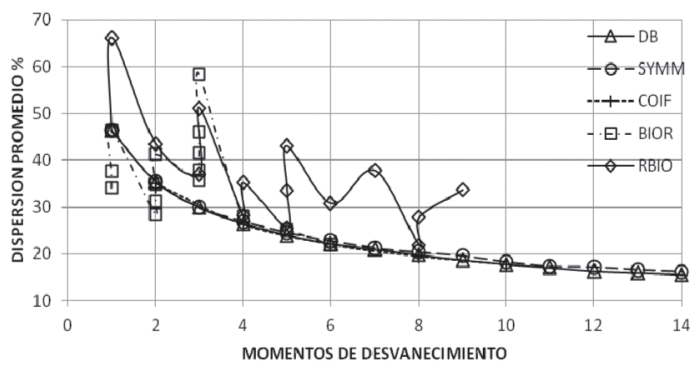

Figura 9. Dispersión promedio de las familias bajo análisis.

Aunque sea lógico inferir que lo más conveniente es usar una wavelet con un mayor número de momentos de desvanecimiento (o alto orden), se debe tener en cuenta que la longitud del filtro aumenta con relación al orden de la wavelet, de modo que a medida que aumenta la cantidad de momentos de desvanecimiento también aumenta el tiempo de cálculo que toma la aplicación de la transformada. Por esto se debe tener un balance entre estas dos propiedades, de tal forma que se seleccione una wavelet que ofrezca poca dispersión de información y requiera un tiempo de cálculo moderado.

En la Figura 10 se grafican los resultados obtenidos pero en función de la longitud del filtro.

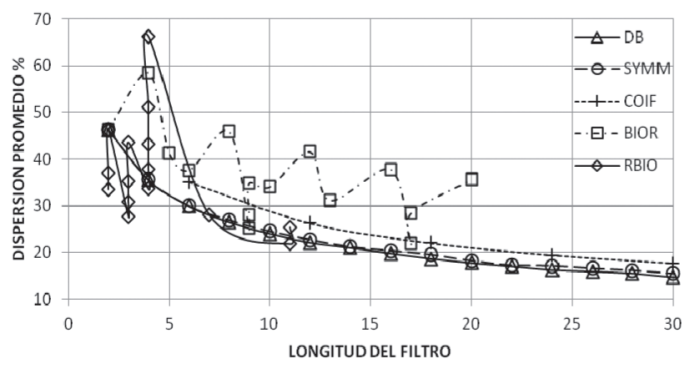

Figura 10. Dispersión promedio en función de la longitud del filtro.

Tomando como referencia a la wavelet db4 [12-14], que como filtro posee ocho coeficientes (Tabla 1), se centra la atención en las wavelets cuya longitud del filtro está entre seis y doce coeficientes. En la Tabla 2 se muestra el grupo de wavelets seleccionadas.
Tabla 2. Grupo de wavelets acotado.

\begin{tabular}{|l|c|c|c|}
\hline Wavelet & $\begin{array}{c}\text { Dispersión } \\
\text { promedio \% }\end{array}$ & $\begin{array}{c}\text { Longitud } \\
\text { del filtro }\end{array}$ & $\begin{array}{c}\text { Momentos de } \\
\text { desvanecimiento }\end{array}$ \\
\hline Db3 & $\mathbf{2 9 , 8 9 5 3}$ & $\mathbf{6}$ & $\mathbf{3}$ \\
\hline Sym3 & 29,8953 & 6 & 3 \\
\hline Coif1 & 35,1067 & 6 & 2 \\
\hline Bior1.3 & 37,5091 & 6 & 1 \\
\hline Rbio4.4 & $\mathbf{2 8 , 1 3 4 6}$ & $\mathbf{7}$ & $\mathbf{4}$ \\
\hline Db4 & $\mathbf{2 6 , 4 9 6 1}$ & $\mathbf{8}$ & $\mathbf{4}$ \\
\hline Sym4 & 26,9376 & 8 & 4 \\
\hline Bior3.3 & 45,9144 & 8 & 3 \\
\hline Bior5.5 & $\mathbf{2 5 , 0 6 4 2}$ & $\mathbf{9}$ & $\mathbf{5}$ \\
\hline Bior4.4 & 28,0054 & 9 & 4 \\
\hline Bior2.4 & 34,7727 & 9 & 2 \\
\hline Db5 & $\mathbf{2 3 , 9 7 5 3}$ & $\mathbf{1 0}$ & $\mathbf{5}$ \\
\hline Sym5 & 24,4773 & 10 & 5 \\
\hline Bior1.5 & 34,0737 & 10 & 1 \\
\hline Rbio6.8 & $\mathbf{2 1 , 8 3 7 9}$ & $\mathbf{1 1}$ & $\mathbf{8}$ \\
\hline Rbio5.5 & 25,3876 & 11 & 5 \\
\hline Db6 & $\mathbf{2 2 , 0 9 7 0}$ & $\mathbf{1 2}$ & $\mathbf{6}$ \\
\hline Sym6 & 22,7049 & 12 & 6 \\
\hline Coif2 & 26,2503 & 12 & 4 \\
\hline Bior3.5 & 41,5002 & 12 & 3 \\
\hline
\end{tabular}

De este grupo se seleccionan las wavelets que ofrecen mejores resultados en la dispersión para cada valor de longitud del filtro: db3, rbio4.4, $\mathrm{db} 4$, bior5.5, db5, rbio6.8 y db6. Se considera la cantidad de momentos de desvanecimiento y el tamaño del soporte de la wavelet para el proceso de selección.

\section{CASO DE APLICACIÓN}

Para validar el método se aplicó el análisis de una señal transitoria obtenida de la suma de diferentes armónicos.

Aplicando la WPD se determinó el valor RMS de la señal a partir de los coeficientes de transformación, utilizando (9) [21-22].

$$
V_{r m s}=\sqrt{\frac{1}{L} \sum_{i} \sum_{k}\left(d_{i}^{j}(k)\right)^{2}}
$$

Donde $L$ es la longitud de la señal digitalizada, $d_{i}^{j}$ es el componente del árbol de descomposición en el nodo $i$ y el nivel $j$. 
Se simuló el transitorio de voltaje de la Figura 11, que tiene una presencia temporal de los armónicos 3,5 y 7 , esto se puede apreciar en la Figura 12 donde se descompone la señal utilizando la WPD.

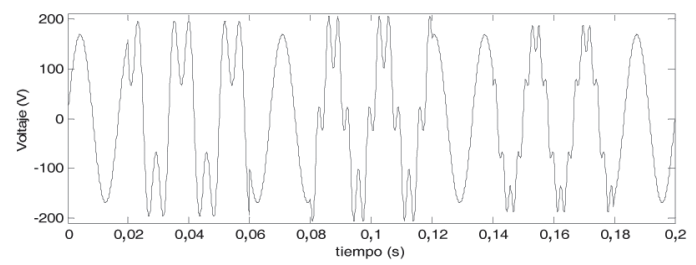

Figura 11. Transitorio de voltaje.
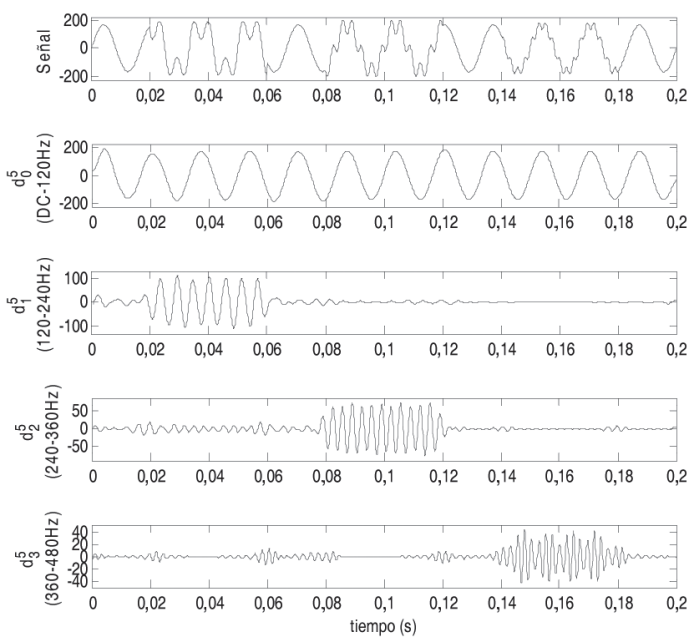

Figura 12. Descomposición por WPD de la señal de la Figura 11.

Con las wavelets seleccionadas en la sección anterior se analizó el transitorio de la Figura 11 con el objeto de encontrar el valor eficaz (RMS) de cada armónico y compararlo con el obtenido de forma analítica; los resultados se presentan en la Tabla 3.

Tabla 3. Valores RMS del transitorio de la Figura 12.

\begin{tabular}{|l|r|r|r|r|c|}
\hline Armónicos & \multicolumn{1}{|c|}{$\begin{array}{c}\mathbf{1} \\
(\mathbf{6 0 H z})\end{array}$} & $\mathbf{3}$ & $\mathbf{5}$ & $\mathbf{7}$ & $\begin{array}{c}\text { Error } \\
\text { promedio } \\
(\mathbf{\%})\end{array}$ \\
\hline Analítico & $119,9 \mathrm{~V}$ & $32,4 \mathrm{~V}$ & $21,4 \mathrm{~V}$ & $10,7 \mathrm{~V}$ & --- \\
\hline Db3 & 2,18 & 26,13 & 2,84 & 5,80 & 9,24 \\
\hline Rbio4.4 & 7,44 & 28,94 & 16,98 & 10,80 & 16,04 \\
\hline Db4 & 0,67 & 7,64 & 4,93 & 7,12 & 5,09 \\
\hline Bior55 & 27,23 & 16,15 & 0,98 & 41,15 & 21,38 \\
\hline Db5 & 0,19 & 5,67 & 9,30 & 10,24 & 6,35 \\
\hline Rbio6.8 & 0,29 & 5,04 & 6,87 & 8,23 & 5,11 \\
\hline Db6 & 0,55 & 4,71 & 0,66 & 3,08 & 2,25 \\
\hline
\end{tabular}

Se observa el buen desempeño de la wavelet db4; sin embargo, para el caso de estudio se selecciona la wavelet db6 debido a que es la que presenta menor error promedio. Se debe tener en cuenta que al utilizar wavelets con un mayor número de momentos de desvanecimiento los resultados mejoran, pero de una forma no muy considerable; pero como los coeficientes del filtro aumentan proporcionalmente a los momentos de desvanecimiento, dicha mejoría en relación con el cálculo computacional no es justificable.

\section{CONCLUSIONES}

El método propuesto permite encontrar una wavelet adecuada para el análisis frecuencial de señales transitorias de acuerdo con sus propiedades, dando mayor importancia a la cantidad de momentos de desvanecimiento (relacionada con la dispersión de información o dispersión de energía de los coeficientes) y al tamaño del soporte (que está relacionado con el tiempo de cálculo), factores relevantes en el análisis frecuencial de señales transitorias.

Con el método propuesto, a partir de un grupo inicial de 100 wavelets se redujo el problema de selección a solo 7 wavelets. Al valorar los resultados obtenidos con el caso de aplicación se ratificó el desempeño de la wavelet $\mathrm{db} 4$, sin embargo, se seleccionó la wavelet db6.

Los resultados del caso de aplicación implementado permitieron validar el método de selección desarrollado tal como se observa en la Tabla 3.

\section{AGRADECIMIENTOS}

Los autores expresan sus agradecimientos a Colciencias por la financiación recibida durante el desarrollo del presente estudio.

\section{REFERENCIAS}

[1] S. Chen and H. Zhu. "Wavelet transform for processing power quality disturbances". EURASIP Journal on Advances in Signal Processing. Vol. 2007, pp. 176-176. January, 2007. ISSN: 1110-8657. DOI: $10.1155 / 2007 / 47695$. 
[2] D. Robertson, O. Camps, J. Mayer and W. Gish. "Wavelets and electromagnetic power system transients". IEEE Transactions on Power Delivery. Vol. 11, pp. 1050-1058. April, 1996. ISSN: 0885-8977. DOI: 10.1109/61.489367.

[3] M. Karimi, H. Mokhtari and M. Iravani. "Wavelet based On-line disturbance detection for power quality applications". IEEE Transactions on Power Delivery. Vol. 15, pp. 1212-1220. October, 2000. ISSN: 08858977. DOI: $10.1109 / 61.891505$.

[4] Z. Leonowicz, T. Lobos and J. Rezmer. "Spectrum estimation of non-stationary signals in power systems". International Conference on Power Systems Transients. New Orleans, USA. September 28-October 2, 2003.

[5] M. Alves. "Transformada em ondeleta: uma necessidade". Revista Brasileira de Ensino de Física. Vol. 28, pp. 563-567. Setembro, 2006. ISSN: 1806-1117. DOI: 10.1590/ S1806-11172006000400019.

[6] E. Schmitt, P. Idowu and A. Morales. "Applications of wavelets in induction machine fault detection". Ingeniare. Revista chilena de ingeniería. Vol. $18 \mathrm{~N}^{\circ} 2$, pp. 158164. Agosto de 2010. ISSN: 0718-3305. DOI: $10.4067 /$ S0718-33052010000200002.

[7] F. Costa, A. Sobrinho, M. Ansaldi and M. Almeida. "The effects of the mother wavelet for transmission line fault detection and classification". Proceedings of the 2011 3rd International Youth Conference on Energetics (IYCE). Leiria, Portugal. July 7-9, 2011.

[8] D. Fugal. "Conceptual Wavelets in digital signal processing". Editorial Space \& Signals Technical Publishing. $1^{\text {st }}$ Edition, p. 374. San Diego, USA. 2009. ISBN: 0982199457.

[9] C. Arizmendi, J. Hernandez-Tamames, E. Romero, A. Vellido and F. del Pozo. "Diagnosis of brain tumours from magnetic resonance spectroscopy using wavelets and Neural Networks". 32nd Annual International Conference of the IEEE Engineering in Medicine and Biology Society, pp. 60746077. Buenos Aires, Argentina. 2010. DOI: 10.1109/ IEMBS.

[10] C. Arizmendi, A. Vellido and E. Romero. "Classification of human brain tumours from MRS data using Discrete Wavelet
Transform and Bayesian Neural Networks". Expert Systems with Applications. Vol. 39, Issue 5, pp. 5223-5232. April, 2012. DOI: 10.1016/ j.eswa.2011.11.017.

[11] E. Rivas, J.C. Burgos and J.C. García-prada. "Condition Assessment of Power OLTC by Vibration Analysis Using Wavelet Transform". IEEE Transactions on Power Delivery. Vol. 24, Issue 2, pp. 687-694. April, 2009.

[12] K. Butler and M. Bagriyanik. "Characterization of transients in transformers using discrete Wavelet transforms". IEEE Transactions on Power Systems. Vol. 18, pp. 648-656. May, 2003. ISSN: 0885-8950. DOI: $10.1109 /$ TPWRS.2003.810979.

[13] Z. Enrang, L. Zhengyan and M. Lingkun. "Study on harmonic detection method based on FFT and Wavelet transform". 2nd International Conference on Signal Processing Systems (ICSPS). Dalian, China. July 5-7, 2010.

[14] P. Purkait and S. Chakravorti. "Pattern classification of impulse faults in transformers by Wavelet analysis". IEEE Transactions on Dielectrics and Electrical Insulation. Vol. 9, pp. 555 -561. August, 2002. ISSN: 10709878. DOI: 10.1109/ TDEI.2002.1024433.

[15] M. Doroslovacki. "Discrete-time signals and uncertainty relations involving ordinary second moments in time and frequency". International Symposium on Time-Frequency and Time-Scale Analysis. Philadelphia, USA. October 25-28, 1994.

[16] S. Mallat. "A Wavelet tour of signal processing". Editorial Elsevier. $3^{\text {rd }}$ Edition, p. 500. United States. 2009. ISBN: 978-0-12-374370-1.

[17] D. Radunovic. "Wavelets from math to practice". Editorial Springer, Academic Mind. Ilustrated Edition, p. 160. Belgrade, Serbia. 2009. ISBN: 9783642006135.

[18] C. Parameswariah and M. Cox. "Frequency characteristics of wavelets". IEEE Transactions on Power Delivery. Vol. 17, pp. 800-804. July, 2002. ISSN: 0885-8977. DOI: 10.1109/TPWRD.2002.1022806.

[19] S. Kelkar, L. Grigsby and J. Langsner. "An extension of Parseval's theorem and its use in calculating transient energy in the frequency domain". IEEE Transactions on Industrial Electronics. Vol. IE-30, pp. 42-45. February, 1983. ISSN: 0278-0046. DOI: 10.1109/ TIE.1983.356702. 
[20] V. Vega, C. Duarte, G. Ordóñez and N. Kagan. "Selecting the best wavelet function for power quality disturbances identification patterns". 13th International Conference on Harmonics and Quality of Power (ICHQP). Wollongong, Australia. September 28-October 1, 2008 .

[21] E. Hamid and Z. Kawasaki. "Wavelet packet transform for RMS values and power measurements". IEEE Power Engineering
Review. Vol. 21, pp. 49-51. September, 2001. ISSN: 0272-1724. DOI: 10.1109/ MPER. 2001.4311610.

[22] R. Diego and J. Barros. "Global method for time-frequency analysis of harmonic distortion in power systems using the wavelet packet transform". Electric Power Systems Research. Vol. 79, pp. 1226-1239. August, 2009. ISSN: 0378-7796. DOI: 10. 1016/j. epsr.2009.03.002. 